\title{
Study on the influence of Alpha wave music on working memory based on EEG
}

\author{
Xin $\mathrm{Xu}^{1}$ and Jiawen Sun ${ }^{1, *}$ \\ ${ }^{1}$ School of Communication and Information Engineering, Nanjing University of Posts and Telecommunications \\ Nanjing, Jiangsu 210003 China \\ [e-mail: sunjwny@163.com] \\ ${ }^{*}$ Corresponding author: Jiawen Sun
}

Received Septebmer 16, 2021; revised November 17, 2021; accepted January 20, 2022; published February 28, 2022

\begin{abstract}
Working memory (WM), which plays a vital role in daily activities, is a memory system that temporarily stores and processes information when people are engaged in complex cognitive activities. The influence of music on WM has been widely studied. In this work, we conducted a series of n-back memory experiments with different task difficulties and multiple trials on 14 subjects under the condition of no music and Alpha wave leading music. The analysis of behavioral data show that the change of music condition has significant effect on the accuracy and time of memory reaction ( $<<0.01)$, both of which are improved after the stimulation of Alpha wave music. Behavioral results also suggest that short-term training has no significant impact on working memory. In the further analysis of electrophysiology (EEG) data recorded in the experiment, auto-regressive (AR) model is employed to extract features, after which an average classification accuracy of $82.9 \%$ is achieved with support vector machine (SVM) classifier in distinguishing between before and after WM enhancement. The above findings indicate that Alpha wave leading music can improve WM, and the combination of AR model and SVM classifier is effective in detecting the brain activity changes resulting from music stimulation.
\end{abstract}

Keywords: Alpha wave leading music, working memory, AR model estimation method, SVM classifier. 


\section{Introduction}

Working Memory (WM) is a memory system that temporarily stores and processes information when people are engaged in complex cognitive activities, such as reasoning and learning. In our daily life, almost all brain activities, including language understanding, decision-making, learning and thinking, involve working memory. Therefore, it is of great practical significance to carry out research on improving working memory. In the research theories of working memory, the well-known "Baddeley Multi-component Model" [1], divides working memory into three sections, involving visual space which is used to store and process visual information, voice loop which is used to store and control information based on sound, and central executive system which is used to link each subsystem with long-term memory, coordinate attention resources, make relevant plans, measure and select optimal strategies. The central executive system is also the core part of working memory.

EEG, which is short for Electroencephalogram, is the general electrical activity of a large number of brain neurons collected by precision electronic instruments on the scalp surface [2]. Compared with other methods that can effectively reflect the working state of the brain, EEG has the advantages of non-radiation and non-invasiveness, which makes it popular in the applied research of analyzing brain decision-making and cognition, and has great practical and social value.

It is known that music has a powerful impact on the brain. Since the 20th century, a large number of scholars have been devoted to researching how music influences brain activities, and they found that music can improve learning efficiency and memory level. However, the details of how music affects the brain remain unclear.

In 1989, the Bulgarian philosopher Lozanov put forward the famous "Suggestive Learning Method" [3]. He used soothing classical music to stimulate brain activity, which improved the concentration and memory. Lozanov argued that soothing music helped to improve memory because the rhythm of 60 beats per minute was coordinated with the rhythm of information input into the brain during learning, which enhanced the alpha wave in brain waves, reduced nervousness and relieved anxiety, thus making the brain more sensitive and stronger in brain activity. Since then, a large body of research works have been carried out based on Lozanov's proposition. In 1995, Rauscher discovered that the spatial memory of the subjects was significantly improved after listening to Mozart music with slow rhythm, which was named "Mozart Effect". Sheila Ostrander [4] also found that under the influence of baroque music, students' learning efficiency increased by $24 \%$, and the memory level increased by $26 \%$. After that, Sun Chang 'an [5] et al. used event-related potentials (ERP) technology to find that Chopin music was beneficial to improve working memory, in which the effect of leading music was the best, followed by background music. Wang Shuaishuai [6] et al. and Li Jipeng [7] et al. used extract low resolution brain electromagnetic tomography (eLORETA), and Li Ying [8] et al. used brain function network analysis, found that rock music and classical music stimulated the brain activity, in which the influence of rock music was more obvious, and background music would occupy the cognitive resources and reduce the working memory ability of the brain. Hu Chengjiang [9] analyzed the influence of music on working memory span with behavioral data, and found that soothing background music was more conducive to improving working memory span than passionate background music. However, the promotion effect of soothing music was not significant, while passionate music has significant interference on working memory span. Lei Min [10] analyzed the influence of background 
music on working memory with behavior data under different rhythms and memory loads, and found that background music with fast rhythm, low volume and that with slow rhythm, high volume improved the working memory accuracy under high memory difficulty, while background music with fast rhythm, high volume and that with slow rhythm, low volume reduced the accuracy. Fast-paced background music improved the reaction speed of WM, while slow-paced background music did the opposite.

In addition to studying whether the difference of music itself has an impact on working memory, some scholars have also conducted research on whether long-term musical training has an impact on WM. Most scholars believe that people who have received long-term musical training have better performance on working memory tasks than people without training. Chen Jiejia [11] used ERP technology to explore the influence of music experience on college students' cognitive flexibility, and found that music experience not only improved the accuracy of WM tasks, but also shortened the response time. By analyzing the behavioral data of the subjects, Anna Marie Fennell et al. [12] found that both musical stimuli and linguistic stimuli interfered with working memory, but visuospatial stimuli did not, indicating that music and language depend on similar WM resources (i.e., verbal skills), while visuospatial depends on different WM resources. Moreover, people with long-term musical training, such as musicians, performed more accurately on working memory tasks, especially in verbal and musical WM stimuli, suggesting a strong correlation between musical ability and verbal working memory ability. Yue D et al. [13] found that musicians performed better than non-musicians in terms of WM capacity and duration when performing the forward tonal discrimination task. In atonal condition, musicians performed better in terms of working memory capacity than non-musicians, but did not perform better in terms of duration.

In the previous studies, ERP analysis, brain network analysis and behavioral data analysis were often used to study the influence of emotion [14] and music on working memory, while AR model estimation method was mostly used in EEG research of thinking pattern classification [15] and central nervous system injury [16]. There was almost no precedent for classifying EEG signals of WM with AR model features. Therefore, we performed a series of n-back EEG memory experiments on 14 subjects with different task difficulties and multiple trials under two different musical conditions: no music and Alpha wave leading music in this study. The research was carried out from two aspects: the behavior data and the EEG feature classification of AR model, in order to explore whether Alpha wave leading music and short-term training impact working memory under different memory loads and whether the AR model are effective for the analysis of working memory.

\section{Materials and Methods}

\subsection{Subjects}

Fourteen participants selected by preliminary experiment were included in the research, including 7 male and 7 female students from non-music majors, aged between 20 and 26. All participants were right-handed, with normal vision or corrected vision and normal auditory function, and they were in good health, without history of mental illness, brain trauma, substance abuse, chronic physical diseases and nervous system diseases. Before the experiment, all the subjects were asked to take a good rest, avoid strenuous exercise and long hours of mental work, informed of the experiment process and volunteered to participate in the experiment. 


\subsection{Experimental materials}

The music material used in the experiment is Alpha wave music, and the memory material is a series of random letters listed according to the 1-back and 2-back rules in n-back tasks. The 1-back task is to judge whether the letter (except the beginning one) displayed on the current screen is consistent with the letter ahead of it. If they are consistent, press the key "1", and if inconsistent, press the key "2", as shown in Fig. 1. The 2-back task is to judge whether the letter (except the beginning two) displayed on the current screen is consistent with the second letter ahead of it. If they are consistent, press the key "1", and if inconsistent, press the key "2", as shown in Fig. 2.

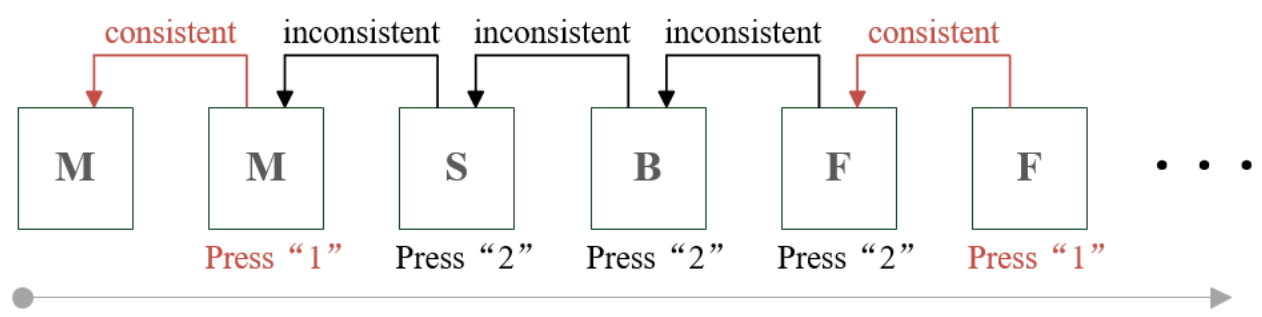

Fig. 1. 1-back experiment task

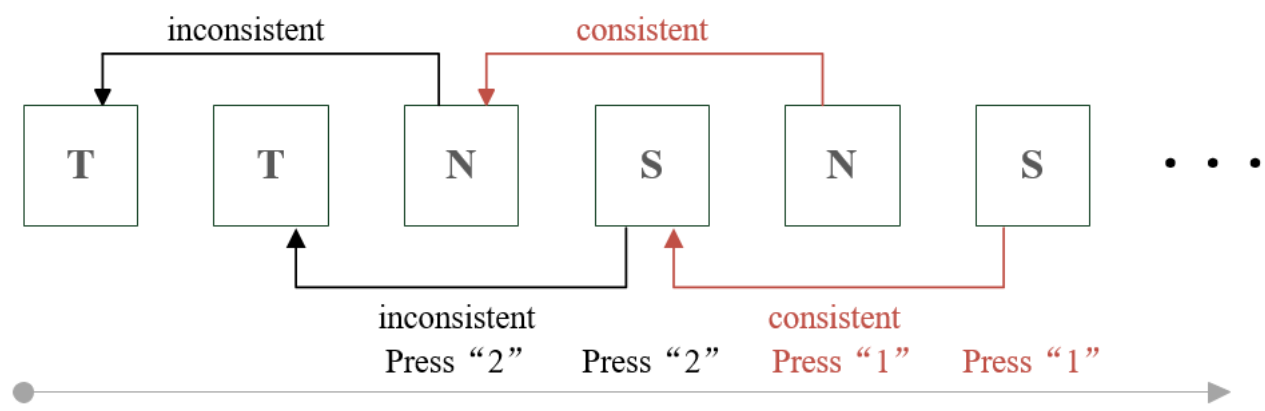

Fig. 2. 2-back experiment task

\subsection{Experimental process}

The experimental process is programmed by E-prime2.0 and designed by 24 in-subject experiment, in which the independent variables are music conditions including no music and Alpha wave leading music, task difficulty including 1-back task with low memory load and 2-back task with high memory load, and experiment times from 1 to 6 . During the experiment, the behavioral responses of the subjects were recorded by E-prime 2.0 and the EEG data were collected by EEG recording instrument.

The experiment was conducted in a quiet laboratory according to the following process. Firstly, the subjects needed to practice the experiment, which consists of two trials, namely 1-back task and 2-back task. The subjects could practice as many times until they were familiar with the whole process and the two tasks. During practice, neither the behavioral data nor the EEG data was recorded. The formal experiment included 6 groups. Each group was divided into 4 trials, including a 1-back task and a 2-back task that were performed in a quiet environment without stimulation and after listening to Alpha wave music. Each task had 20 stimuli excluding the first one letter in the 1-back task and the first two letters in the 2-back task. The presentation time of each stimulus was $1500 \mathrm{~ms}$, during which the subjects were required to react according to the task rules. The stimulation interval was $1000 \mathrm{~ms}$, during 
which the subjects had to keep their memory. The Alpha wave music was played for 1 minute, during which the subjects were required to close their eyes, stay relaxed and throw themselves into the music. Each group of the experiments lasted about 6 to 7 minutes, after which the subjects would take a rest before the next group. Fig. $\mathbf{3}$ is the flowchart of a group of formal experiments.
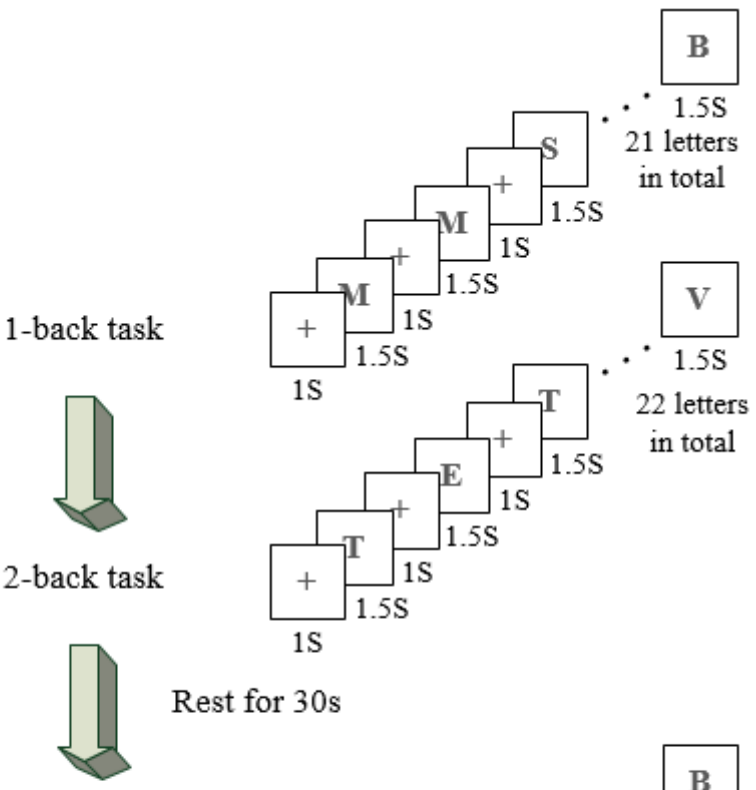

Close the eyes, listen to music for 1 minute

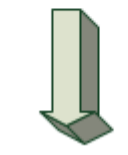

1-back task
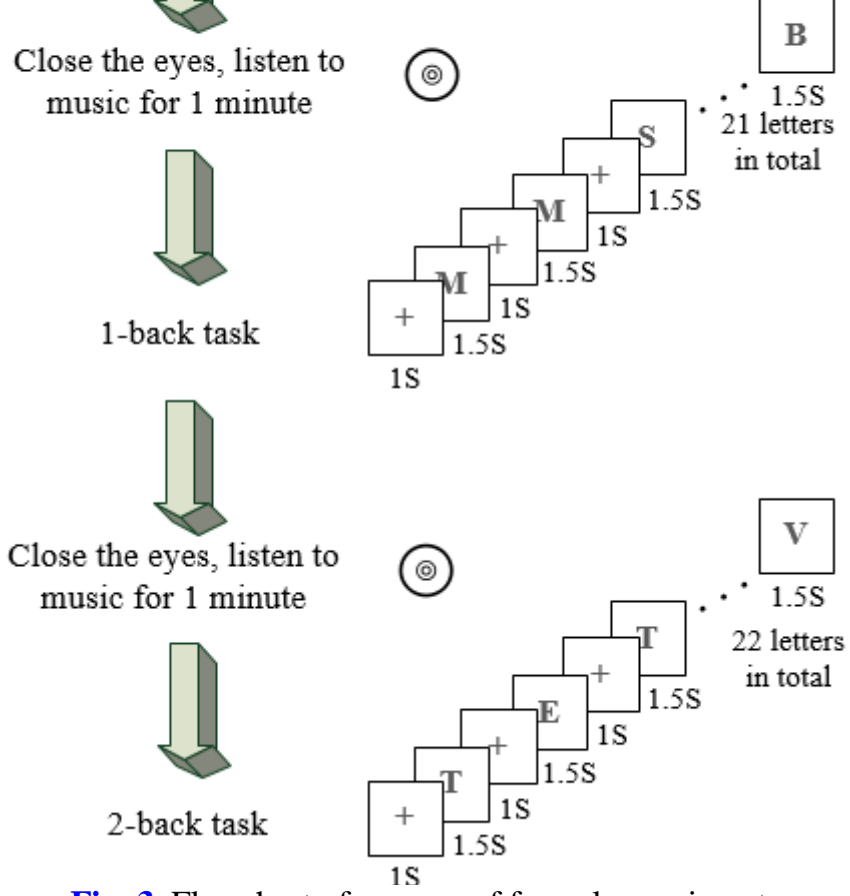

Fig. 3. Flowchart of a group of formal experiments 


\subsection{Data acquisition and processing}

During the experiment, E-prime 2.0 was used to record behavioral data, including the reaction results and response time of the subjects, so as to analyze the behavior data with SPSS. The EEG acquisition equipment used in the experiment was the EEG cognitive potential equipment produced by Nanjing Weisi Company, and a 19-lead electrode cap taking the bilateral mastoid as the reference electrode. The impedance between the scalp and the electrode was required to be less than $30 \mathrm{~K} \Omega$. The filter was low-pass $30 \mathrm{~Hz}$ and the sampling rate was 512Hz / lead. After repeated screening, 13 channels including Fp1, Fp2, F4, C3, C4, P3, P4, O1, O2, T3, T4, Fz and Pz were selected in the experiment, as shown in Fig. 4.

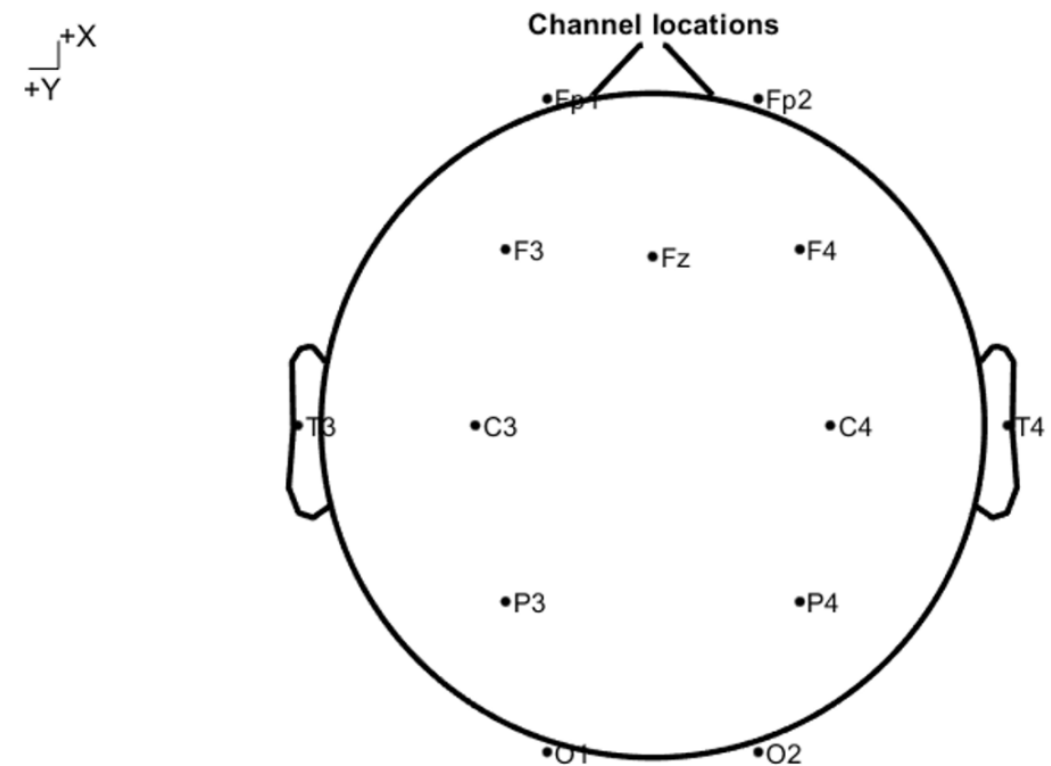

Fig. 4. Map of the 13 channels selected in the experiment

Each subject performed 6 groups of formal experiments, and each group of formal experiments was recorded as a segment of EEG data. At the beginning of each formal experiment, the subject must press the start button on E-Prime which automatically recorded each time point of the start and response. Meanwhile, the subject also pressed the EEG data recording button. When processing the EEG data after the experiment, we marked the response events in the recorded EEG data, and then intercepted the data of 0 to 1 second from the appearance of each letter that needed to be responded to. Since a formal experiment consists of four trials, each of which has 20 letters to respond to, a formal experiment includes 80 1-second EEG signal under 13 channels.

Due to the characteristics of high randomness and event sensitivity of EEG signals, the collected signals are usually mixed with noise and technical or biological artifacts, such as power line interfere, eyeblink and muscle activity. In order to reduce the interference of artifacts on EEG signal processing, before EEG data was processed formally, independent component analysis (ICA) algorithm in EEGLAB was used to preprocess the EEG signals. 


\section{Data analysis and results}

\subsection{Behavior data processing}

A 1-back or 2-back task is taken as a sample, which has 20 reaction results and 20 reaction time differences. Before processing, those that fail to respond are counted as error responses in the accuracy statistics, and infinite response times in the response time statistics. Then, the original behavior data above are processed as follows. Firstly, divide the correct response amount by the total amount of 20 in each sample to get the accuracy rate. Secondly, take the median of the reaction time differences in each sample to get the response time.

We perform multivariate variance analysis on music condition, task difficulty and experimental times using SPSS. Statistical results show that: (1) music condition had a significant relationship with accuracy $(\mathrm{p}<0.01)$, and a general significant relationship with response time $(\mathrm{p}<0.1),(2)$ task difficulty has a significant relationship with both accuracy and reaction time $(\mathrm{p}<0.01)$, (3) experimental times has a significant relationship with reaction time $(\mathrm{P}<0.01)$, but no significant effect on accuracy, and (4) the interaction effect among music conditions, task difficulty and experimental times on reaction time and accuracy are insignificant. The test results of inter-subject effect are shown in Table 1, and the unit of reaction time in this paper is $\mathrm{ms}$. In the following tables, * means $\mathrm{P}<0.1$, ** means $\mathrm{P}<0.05$, *** means $\mathrm{P}<0.01$.

Table 1. Inter-subject effect test

\begin{tabular}{|c|c|c|c|c|c|c|}
\hline $\begin{array}{c}\text { Independent } \\
\text { variable }\end{array}$ & $\begin{array}{c}\text { Dependent } \\
\text { variable }\end{array}$ & $\begin{array}{c}\text { Class III sum } \\
\text { of squares }\end{array}$ & Freedom & Mean square & F & Significance \\
\hline \hline \multirow{2}{*}{ Musical conditions } & Reaction time & 45198.241 & 1 & 45198.241 & 3.104 & $0.079 *$ \\
\cline { 2 - 7 } & Accuracy & 0.034 & 1 & 0.034 & 8.512 & $0.004^{* * *}$ \\
\hline \multirow{2}{*}{ Task difficulty } & Reaction time & 956373.441 & 1 & 956373.441 & 65.669 & $0.000^{* * *}$ \\
\cline { 2 - 7 } & Accuracy & 0.122 & 1 & 0.122 & 30.160 & $0.000 * * *$ \\
\hline \multirow{2}{*}{\begin{tabular}{c} 
experimental times \\
\cline { 2 - 7 }
\end{tabular}} & Reaction time & 746187.792 & 5 & 149237.558 & 10.247 & $0.000 * * *$ \\
\hline \multirow{2}{*}{$\begin{array}{c}\text { Music conditions * } \\
\text { task difficulty }\end{array}$} & Accuracy & 0.036 & 5 & 0.007 & 1.763 & 0.120 \\
\cline { 2 - 7 } & Accuracy & 0.001 & 1 & 0.001 & 0.265 & 0.607 \\
\hline $\begin{array}{c}\text { Music conditions * } \\
\text { experimental times }\end{array}$ & Reaction time & 9882.821 & 5 & 1976.564 & 0.136 & 0.984 \\
\cline { 2 - 7 } & Accuracy & 0.021 & 5 & 0.004 & 1.024 & 0.404 \\
\hline $\begin{array}{c}\text { Task difficulty * } \\
\text { experimental times }\end{array}$ & Reaction time & 20833.265 & 5 & 4166.653 & 0.286 & 0.921 \\
\cline { 2 - 7 } & Accuracy & 0.009 & 5 & 0.002 & 0.423 & 0.833 \\
\hline $\begin{array}{c}\text { Music conditions * } \\
\text { task difficulty * } \\
\text { experimental times }\end{array}$ & Reaction time & 11437.723 & 5 & 2287.545 & 0.157 & 0.978 \\
\cline { 2 - 8 } & Accuracy & 0.006 & 5 & 0.001 & 0.278 & 0.925 \\
\hline
\end{tabular}

According to the significant results in Table 1, the effects of music conditions, task difficulty and experiment times on reaction time and accuracy are further analyzed. The estimated average values at the 95\% confidence interval are shown in Table 2. 
Table 2. Estimated average value

\begin{tabular}{|c|c|c|c|c|}
\hline $\begin{array}{c}\text { Independent } \\
\text { variable } \\
\end{array}$ & $\begin{array}{c}\text { Dependent } \\
\text { variable } \\
\end{array}$ & Situations & Average value & $\begin{array}{c}\text { Standard } \\
\text { error }\end{array}$ \\
\hline \multirow{4}{*}{$\begin{array}{c}\text { Musical } \\
\text { conditions }\end{array}$} & \multirow[b]{2}{*}{ Reaction time } & No music & 632.601 & 9.311 \\
\hline & & $\begin{array}{c}\text { Alpha wave } \\
\text { music }\end{array}$ & 609.405 & 9.311 \\
\hline & \multirow[b]{2}{*}{ Accuracy } & No music & 0.938 & 0.005 \\
\hline & & $\begin{array}{c}\text { Alpha wave } \\
\text { music }\end{array}$ & 0.958 & 0.005 \\
\hline \multirow{4}{*}{ Task difficulty } & \multirow{2}{*}{ Reaction time } & easy & 567.652 & 9.311 \\
\hline & & difficult & 674.354 & 9.311 \\
\hline & \multirow{2}{*}{ Accuracy } & easy & 0.967 & 0.005 \\
\hline & & difficult & 0.929 & 0.005 \\
\hline \multirow{12}{*}{$\begin{array}{l}\text { Experiment } \\
\text { times }\end{array}$} & \multirow{6}{*}{ Reaction time } & 1 & 700.723 & 16.126 \\
\hline & & 2 & 664.554 & 16.126 \\
\hline & & 3 & 603.179 & 16.126 \\
\hline & & 4 & 610.393 & 16.126 \\
\hline & & 5 & 582.116 & 16.126 \\
\hline & & 6 & 565.054 & 16.126 \\
\hline & \multirow{6}{*}{ Accuracy } & 1 & 0.938 & 0.008 \\
\hline & & 2 & 0.935 & 0.008 \\
\hline & & 3 & 0.948 & 0.008 \\
\hline & & 4 & 0.960 & 0.008 \\
\hline & & 5 & 0.946 & 0.008 \\
\hline & & 6 & 0.963 & 0.008 \\
\hline
\end{tabular}

As shown in Table 2, after listening to Alpha wave music, the response time of the subjects became shorter and the accuracy became higher. The more difficult the task is, the longer the response time and the lower the accuracy. The more times of experiments, the shorter the response time, while no significant change in accuracy.

\subsection{EEG data processing}

\subsubsection{EEG feature extraction}

Autoregressive Model, AR model for short, is applied to extract features from EEG data. The basic idea of AR model for feature extraction is to use AR model to approximate EEG signals. As a linear prediction model, AR model predicts the random variables at a certain time in the later period based on the data of random variables at many moments in the early stage, as shown in (1). Therefore, when the parameters and order of AR model are properly selected, the AR process can be as consistent as possible with the real EEG signal.

$$
x(n)=-\sum_{i=1}^{p} a_{p}(i) x(n-i)+\varepsilon(n)
$$

Where $\varepsilon(n)$ is a white noise sequence with a mean value of zero and a variance of $\sigma^{2}$, and $p$ is the order of AR model. Some of well-known methods to estimate AR model coefficients include autocorrelation method, covariance method, solving Yule-Walker equation method such as Burg algorithm, Levinson-Durbin recursion method, etc. In this paper, Burg algorithm is used to solve AR model parameters.

The goal of Burg algorithm is to minimize the average power of forward prediction error and backward prediction error, as shown in (2). 


$$
\rho^{f_{0}}=\frac{1}{2}\left[\rho^{f}+\rho^{b}\right]
$$

The expressions of forward prediction error power $\rho^{\mathrm{f}}$ and backward error power $\rho^{\mathrm{b}}$ are respectively shown in (3) and (4).

$$
\begin{aligned}
\rho^{f} & =\frac{1}{M-p} \sum_{n=p}^{M-1}\left|e_{p}^{f}(n)\right|^{2} \\
\rho^{b} & =\frac{1}{M-p} \sum_{n=p}^{M-1}\left|e_{p}^{b}(n)\right|^{2}
\end{aligned}
$$

Where $M$ is the data length, $e_{p}^{f}(n)$ is the forward prediction error, and $e_{p}^{b}(n)$ is the backward prediction error. According to the recursion relation, we can derive two relations as shown in (5) and (6).

$$
\begin{gathered}
e_{p}^{f}(n)=e_{p-1}^{f}(n)+k_{p} e_{p-1}^{b}(n-1) \\
e_{p}^{b}(n)=e_{p-1}^{b}(n)+k_{p}^{*} e_{p-1}^{f}(n)
\end{gathered}
$$

The AR model features selected in the experiment are the original data power and the prediction error power of each order, the number of which is 6 in the experiment. Therefore, 7 features can be obtained from the EEG data of every $1 \mathrm{~s}$ in each channel. The letters that need to be reacted are taken as a sample, and each sample contains $13 \times 7=91$ features.

\subsubsection{Classification of EEG characteristics}

The experiment divides the data sets into four categories: (1) low difficulty with no music, (2) low difficulty with Alpha leading music, (3) high difficulty with no music, and (4) high difficulty with Alpha leading music. Each type of dataset has 14 subjects $\times 6$ trails $\times 20$ letters $=1680$ samples.

We classify EEG data before and after Alpha wave music stimulation under the following 3 conditions: low difficulty memory tasks, high difficulty memory tasks, and mixing high and low difficulty memory tasks.

Due to the small sample size, five-fold cross validation is used where the datasets are divided into five heaps. One heap is selected as the test set, and the other four heaps as the training set, which is repeated for five times. We compare the classification results using Naïve Bayes, Linear Discriminant Analysis (LDA), k-nearest neighbor classifier (KNN) with polynomial kernel, Bagged Trees, Boosted Trees, Random Forest, Support Vector Machine (SVM) with polynomial and Gaussian kernel. The classification accuracy of the eight classifiers is shown in Table $\mathbf{3}$ and the statistical diagram is shown in Fig. 5. It can be clearly seen from the figure that SVM with polynomial kernel has the highest classification accuracy, followed by Bagged Trees, SVM with Gaussian kernel, KNN, Boosted Trees, LDA, Random Forest and Naïve Bayes. Therefore, SVM with polynomial kernel is finally selected to classify the extracted features.

Table 3. Classification accuracy of eight classifiers

\begin{tabular}{|c|c|c|c|c|c|c|c|c|}
\hline & $\begin{array}{c}\text { Naïve } \\
\text { Bayes }\end{array}$ & LDA & KNN & $\begin{array}{c}\text { Bagged } \\
\text { Trees }\end{array}$ & $\begin{array}{c}\text { Boosted } \\
\text { Trees }\end{array}$ & $\begin{array}{c}\text { Random } \\
\text { Forest }\end{array}$ & $\begin{array}{c}\text { Gaussian } \\
\text { SVM }\end{array}$ & $\begin{array}{c}\text { Polynomial } \\
\text { SVM }\end{array}$ \\
\hline \hline 1-back task & $59.8 \%$ & $66.8 \%$ & $76.7 \%$ & $79.8 \%$ & $66.5 \%$ & $64.8 \%$ & $78.8 \%$ & $80.3 \%$ \\
\hline 2-back task & $60.5 \%$ & $65.1 \%$ & $80.4 \%$ & $81.6 \%$ & $70.3 \%$ & $66.6 \%$ & $82.0 \%$ & $82.3 \%$ \\
\hline total & $59.6 \%$ & $65.7 \%$ & $80.0 \%$ & $82.4 \%$ & $65.9 \%$ & $62.9 \%$ & $81.6 \%$ & $82.9 \%$ \\
\hline
\end{tabular}




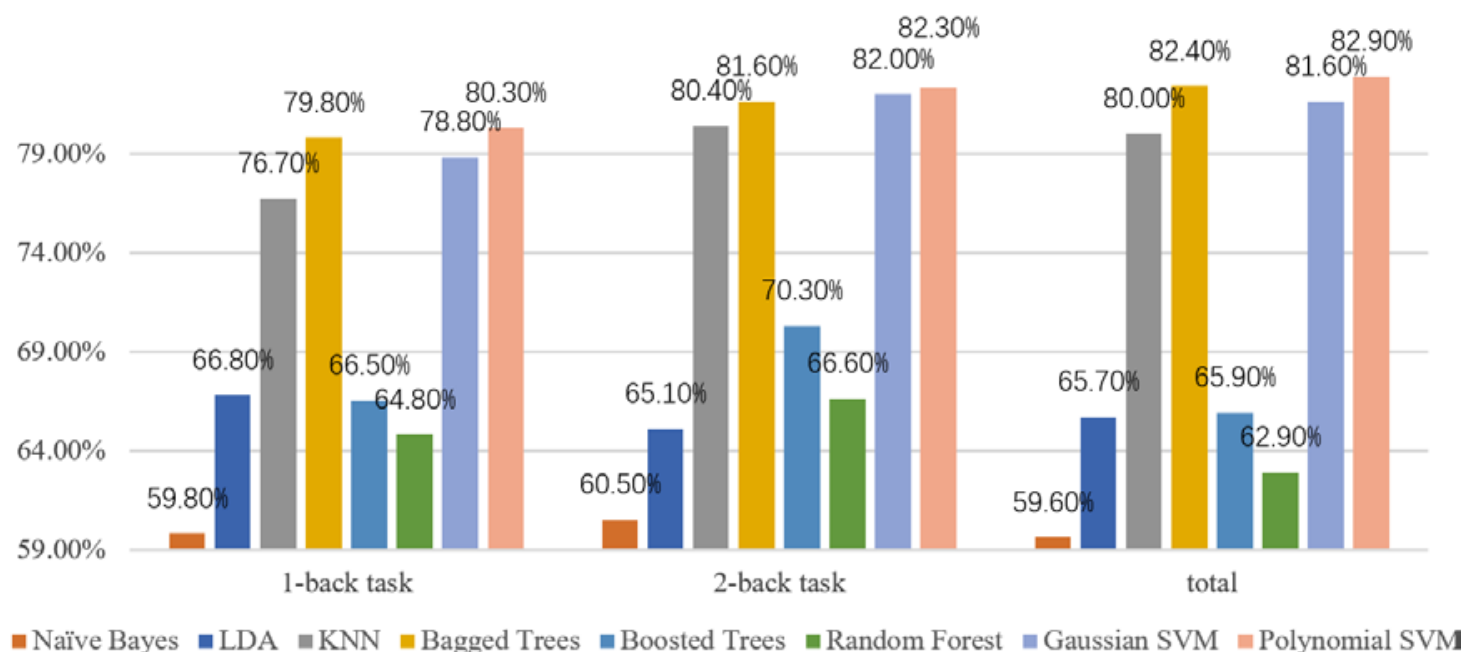

Fig. 5. Statistical diagram of the classification accuracy of eight classifiers

SVM is a typical dichotomous supervised learning algorithm, which was first proposed by Cortes et al. in 1995 [17]. It is widely used because it has certain advantages in solving small sample, nonlinear and high dimensional pattern recognition, and has the characteristics of high recognition accuracy and high speed. The goal of SVM classifier is to find an optimal hyperplane that maximizes the margin between classes. For example, the two-dimensional plane shown in Fig. 6 has two different kinds of data, one with a circle and the other with a cross. Since these data are linearly separable, they can be separated by a straight line, which is the equivalent of a hyperplane.

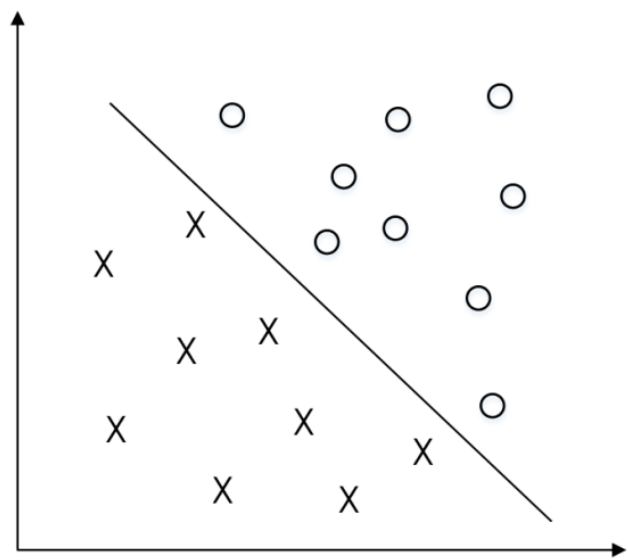

Fig. 6. Linear classification of SVM

However, in practice, we can't find a linear separable hyperplane to achieve effective classification, so in the case of nonlinear, we often choose a kernel function of SVM to make the data divided by mapping the linear indivisible data in the original space to a high-dimensional feature space. In this experiment, the common Polynomial Kernel and Gaussian Kernel are selected. 
Polynomial kernel function, as shown in (7), has many parameters, so when the order of polynomial is large, the value of kernel matrix will tend to infinity or infinitesimal, and the computational complexity will be too large to be calculated. Therefore, the third-order polynomial is chosen in the experiment.

$$
k\left(x, x_{i}\right)=\left(\left(x \cdot x_{i}\right)+1\right)^{d}
$$

Gaussian kernel function, as shown in (8), is the most widely used. While constructing a model, the smaller the distance between two vectors is, the larger the result of the Gaussian kernel function will be.

$$
k\left(x, x_{i}\right)=\exp \left(-\frac{\left\|x-x_{i}\right\|^{2}}{\delta^{2}}\right)
$$

\section{Discussion}

The results of behavioral data analysis show that the response accuracy of the subjects under different music conditions ( $\mathrm{F}=8.512, \mathrm{P}=0.004)$ and task difficulty $(\mathrm{F}=30.160, \mathrm{P}=0.000)$ are significantly different. The response time under different music conditions ( $F=3.104$, $\mathrm{P}=0.079)$, task difficulty $(\mathrm{F}=65.669, \mathrm{P}=0.000)$ and multiple experimental trials $(\mathrm{F}=10.247$, $\mathrm{P}=0.000$ ) are also significantly different. Meanwhile, behavioral data shows that experimental times have no significant effect on response accuracy, and the interaction effect among music conditions, task difficulty and experimental times on accuracy and response time is not significant. Under the condition of no music, the average reaction accuracy is $93.8 \%$ plus or minus $0.5 \%$, and the average reaction time is $632.601 \mathrm{~ms}$ plus or minus $9.311 \mathrm{~ms}$, while after Alpha wave music stimulation, the average accuracy and reaction time are $95.8 \%$ plus or minus $0.5 \%$ and $609.405 \mathrm{~ms}$ plus or minus $9.311 \mathrm{~ms}$ respectively.

The results of behavioral data indicate that the accuracy of the subjects is significantly improved and the response time is significantly shortened after Alpha wave music stimulation, which shows that Alpha wave music can improve working memory. Each subject has gone through six groups of experiments, and with the advancement of group experiment, the reaction time of the experiment has been significantly shortened. However, the accuracy has not changed significantly, which indicates that short-term training improves the sensitivity and proficiency of the subjects, but does not have a significant effect on working memory. Therefore, combining the behavioral data and the EEG data of the six groups of experiments will not have a significant impact on the experimental results. In addition, the accuracy of high-difficulty tasks is significantly lower than that of low-difficulty tasks, and the response time of high-difficulty tasks is significantly longer than that of low-difficulty tasks. The results indicate that, between the two tasks, the low-difficulty tasks need less memory resources when memorizing and refreshing working memory than the high-difficulty tasks. But when the music conditions and task difficulties work together, there is no significant impact on the accuracy and response time, indicating that Alpha wave music stimulation has no obvious difference in improving working memory when completing memory tasks with different difficulties. In conclusion, Alpha wave leading music can improve working memory, but has no significant effect on the improvement degree of different cognitive loads.

In the process of EEG data analysis, we extract AR model features of EEG signals and analyze EEG signals when working memory tasks are completed before and after Alpha wave music stimulation. Then we classify the extracted features by SVM classification method and 
the total classification accuracy is $82.9 \%$ with five-fold cross-validation.

In the previous studies, most researchers judge the changes of working memory by analyzing the P300 component in ERP combined with behavioral data and they think that the more the brain resources put into working memory tasks, the higher the amplitude of P300 component [14]. For example, Sun Chang 'an [5] et al. observed the amplitude of P300 component in EEG signals under the conditions of leading music, background music and no music, and found that the amplitude of this component showed a decreasing trend. Combined with the analysis results of its behavior data, it was concluded that leading music was helpful to improve working memory. In this paper, AR model feature is selected to classify EEG signals before and after WM improvement, because the mathematical statistical characteristics derived from AR model estimation method can effectively extract the P300 components in EEG signals [18]. Therefore, behavioral analysis combined with EEG classification results shows that the combination of AR model estimation and SVM classifier is effective to classify EEG signals before and after WM improvement.

\section{Conclusion}

This paper shows that Alpha wave leading music can improve working memory, and the effects are consistent across different memory loads. In addition, short-term training has no significant effect on working memory. At present, in the research of improving working memory, ERP analysis is widely used, but the process of ERP analysis is complex, which has to analyze various components of ERP using human eyes. In this work, AR model and SVM are combined to classify WM before and after the improvement, the result of which is satisfactory, which shows that AR model combined with SVM classification is effective in judging whether working memory is improved or not. This work provides a new method for exploring the improvement of working memory.

\section{References}

[1] A. Baddeley, Working Memory, Thought, and the Action, Oxford, UK: Oxford University Press, 2007. Article (CrossRef Link)

[2] M. Hallett, "Transcranial magnetic stimulation and the human brain," Nature, vol. 406, no. 6792, pp. 147-150, July. 2000. Article (CrossRef Link)

[3] X. Wang, M. B. Zhong, "Variation characteristics and analysis of 'Mozart effect' based on EEG", Journal of Liaocheng University (Natural Science Edition), vol. 17, no. 2, pp. 104-105,107, June, 2004. Article (CrossRef Link)

[4] S. Ostrander, Super Learning Method 2000, Peking, CHINA: China Drama Publishing House, 2001.

[5] C. A. Sun, H. T. Wei, L. J. Yue, "ERP Study on the Influence and Mechanism of Music on Working Memory,” Psychological and Behavioral Research, vol. 11, no. 2, pp. 195-199, 2013. Article (CrossRef Link)

[6] S. S. Wang, Y. Li, J. P. Li, et al, "Research on the effect of background music on spatial cognitive working memory based on cortical brain network," Journal of Biomedical Engineering, vol. 37, no. 4, pp. 587-595, 2020. Article (CrossRef Link)

[7] J. P. Li, Y. Li, D. Y. Zhang, et al, "Research on the Influence of Music Type on Learning and Memory Based on EEG Signal Source Tracing Analysis," Chinese Journal of Biomedical Engineering, vol. 38, no. 6, pp.679-686, June. 2019. Article (CrossRef Link) 
[8] Y. Li, D. Y. Zhang, Q. Su, et al, "Research on the Influence of Classical Music and Rock Music on Working Memory Based on the Brain Network," Chinese Journal of Biomedical Engineering, vol. 38, no. 2, pp. 129-137, Feb. 2019. Article (CrossRef Link)

[9] C. J. Hu, "An experimental study on the relationship between background music and attention, working memory and learning efficiency,” M.S. thesis, Dept. Edu, QNU, Xining, Qinghai, China, 2017. Article (CrossRef Link)

[10] M. Lei, "The influence of background music on working memory,” M.S. thesis, Dept. Edu, ZZU, Zhengzhou, Henan, China, 2016. Article (CrossRef Link)

[11] J. J. Chen, "The effect of music speed on inhibitory control: behavioral and EEG research,” M.S. thesis, Dept. Cognitive Neuroscience, HNU, Changsha, Hunan, China, 2020. Article (CrossRef Link)

[12] A. M. Fennell, J. A. Bugos, B. R. Payne, et al, "Music is similar to language in terms of working memory interference,” Psychonomic Bulletin \& Review, vol. 28, no. 2021, pp. 512-525, 2021. Article (CrossRef Link)

[13] D. Yue, G. Kathleen, F. Alexander, et al, "A behavioral study on tonal working memory in musicians and non-musicians,” PLoS ONE, vol. 13, no. 8, pp. 1-18, Aug. 2018. Article (CrossRef Link)

[14] X. Liu, S. Liu, D. Guo, Y. Sheng, D. Ming, "Effect of Emotion States on the Updating Function of Working Memory," in Proc. of 40th Annual International Conference of the IEEE Engineering in Medicine and Biology Society (EMBC), Hawaii, USA, 2018. Article (CrossRef Link)

[15] H. P. Jia, "Classification for EEG signals of different mental tasks," Electronic Design Engineering, vol. 18, no. 6, pp. 118-120, June. 2010. Article (CrossRef Link)

[16] T. S. Qiu, H. Y. Wang, H. P. Bao, et al, “AR model based injury detection of the central nervous system with EEG signals,” International Journal of Biomedical Engineering, vol. 25, no. 2, pp. 92-96, Feb. 2002. Article (CrossRef Link)

[17] C. Cortes, V. Vapnik, “Support-vector networks,” Machine learning, vol. 20, no. 3, pp. 273-297, 1995. Article (CrossRef Link)

[18] L. Huang, R. Li, J. Gu, "EEG Signals Classification Based on AR Model and SVM Algorithm," Neural Networks, vol. 31, no. 35, pp. 24-27, 2013. Article (CrossRef Link)

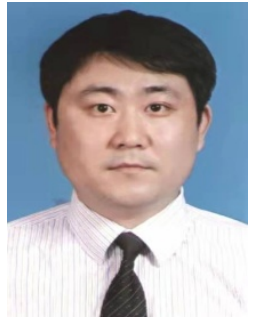

Xin Xu received the master's degree from Nanjing University. He is currently pursuing the Ph.D. degree with the Nanjing University of Science and Technology. He joined the Nanjing University of Posts and Telecommunications, in August 2000. He was promoted to an Associate Professor, in May 2012. He has been a Visiting Scholar with Hong Kong Polytechnic University, since October 2018. He has published about 30 articles. He holds three patents. His research interests include signal and information processing, artificial intelligence, bioelectrical signal analysis and processing and their applications.

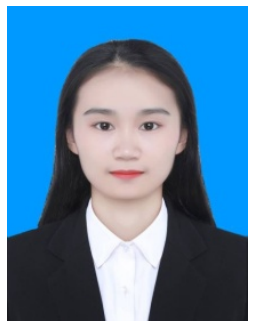

Jiawen Sun is currently pursuing the master's degree in the Nanjing University of Posts and Telecommunications (NUPT). Her research interests include signal and information processing, bioelectrical signal analysis, processing and their applications. 